\title{
A small interfering RNA targeting the KLF6 splice variant, KLF6-SV1, as gene therapy for gastric cancer
}

\author{
Hui Chen • Lili Chen • Lingyu Sun • \\ Hongqun Zhen $\cdot$ Xiaomei Li • Qifan Zhang
}

Received: 5 December 2010/ Accepted: 28 March 2011/Published online: 3 May 2011

(c) The International Gastric Cancer Association and The Japanese Gastric Cancer Association 2011

\begin{abstract}
Background Accumulating evidence suggests that the tumor suppressor gene Kruppel-like factor 6 (KLF6) and its dominant-negative splice form KLF6-SV1 play important roles in both the development and progression of cancer. However, the role of KLF6-SV1 in gastric cancer remains largely unknown.

Methods KLF6-SV1 expression was detected in various human gastric cancer cell lines and gastric cancer patient samples by reverse transcriptase polymerase chain reaction (RT-PCR) and Western blotting. Small interfering RNA (siRNA) was used to inhibit KLF6-SV1 expression in BGC-823 and SGC-7901 cell lines. The effects of downregulation of KLF6-SV1 by siRNA on cell proliferation, migration, invasion, and tumor growth were examined in vitro and in vivo.
\end{abstract}

H. Chen, L. Chen, and L. Sun contributed equally to this work.

H. Chen $\cdot$ X. Li

Department of General Surgery, Third Affiliated Hospital

of Harbin Medical University, 150040 Harbin,

People's Republic of China

L. Chen

Department of General Surgery, Second Affiliated Hospital

of Harbin Medical University, 150040 Harbin,

People's Republic of China

L. Sun $\cdot$ H. Zhen $\cdot$ Q. Zhang

Department of General Surgery, Fourth Affiliated Hospital of Harbin Medical University, 150040 Harbin,

People's Republic of China

Q. Zhang ( $\square)$

Department of General Surgery, Tumor Hospital of Harbin Medical University, 150040 Harbin, People's Republic of China e-mail: qfzhang10@gmail.com
Results Overexpression of KLF6-SV1 was detected in tumor samples from gastric cancer patients, and in various differentiated gastric cancer cell lines. In vitro downregulation of KLF6-SV1 by siRNA inhibited BGC-823 and SGC-7901 cell proliferation, anchorage-independent growth, migration, and invasion through the altered expression of $\mathrm{Ki}-67$, vascular endothelial growth factor (VEGF), E-cadherin, and matrix metalloproteinase (MMP)9. Also, KLF6-SV1 silencing promoted caspase-dependent apoptosis of BGC-823 and SGC-7901 cells via the regulation of phosphatidylinositol 3-OH kinase (PI3K)/Akt activity and Bcl-2-related protein expression. In vivo animal studies showed that KLF6-SV1 siRNA significantly inhibited the tumorigenicity of BGC-823 and SGC-7901 cells. Gene therapy with polyethylenimine/si-SV1 intratumoral injection also resulted in the suppression of tumor growth and prolonged animal survival in an established xenograft tumor model.

Conclusion These data demonstrate that KLF6-SV1 is an important regulator of the growth, migration, invasion, and survival of gastric cancer cells, and downregulation of KLF6-SV1 by siRNA may offer a new potential gene therapy approach for gastric cancer.

Keywords Gastric cancer $\cdot$ KLF6-SV1 - RNA interference · Apoptosis · Tumor gene therapy

\section{Introduction}

Gastric cancer remains a leading cause of cancer mortality, despite a worldwide decline in its incidence. In Asian countries, gastric cancer is one of the most prevalent tumors and is the leading cause of cancer death [1]. In the Western world, more than $80 \%$ of gastric cancer patients have 
advanced cancer on diagnosis, with poor prognosis [2]. Despite the use of multimodal therapy (chemotherapy, radiation therapy, and surgery), the long-term disease-free survival rate in gastric cancer patients is still disappointingly low, particularly in the high-risk groups [1,2]. Although many well-characterized tumor suppressor genes and oncogenes have been defined, along with their respective roles in gastric cancer development and progression [3], the identification of new therapeutic targets is still urgent.

Recent evidence suggests that the tumor suppressor gene Kruppel-like factor 6 (KLF6; also known as COPEB and ZF9) plays a critical role in the development and progression of cancer through regulating cancer cell proliferation, invasion, migration, and survival [4]. Functional inactivation of the KLF6 gene, including loss of heterozygosity (LOH), somatic mutation, and/or increased alternative splicing, has been implicated in a number of human cancers, including prostate [5, 6], colorectal [7, 8], nonsmall cell lung [9, 10], gastric [11], glioma [12, 13], nasopharyngeal [14], hepatocellular [15-18], pancreatic [19], and ovarian carcinomas [20]. To date, three alternatively spliced KLF6 isoforms, KLF6-SV1, -SV2 and -SV3, have been identified [21]. KLF6-SV1 is an oncogenic splice variant of KLF6, and increased KLF6-SV1 expression is associated with poor prognosis in prostate, lung, and ovarian cancers [10, 13, 21-23]. Furthermore, KLF6-SV1 has been shown to be biologically active, antagonizing the tumor suppressor function of KLF6 and promoting tumor growth and dissemination in both ovarian and prostate cancer models [20, 21, 23]. Evidence from many cancer types and different stages of tumorigenesis and progression has confirmed important roles for KLF6 and KLF6-SV1; in particular, that the overexpression of KLF6-SV1 is associated with metastatic potential and with decreased survival and that small interfering RNA (siRNA)-mediated downregulation of KLF6-SV1 decreases tumor growth in vitro [20, 21] and in vivo [10, 23]. In addition, KLF6-SV1 has been identified as a novel anti-apoptotic protein, independent of a tumor cell's p53 status [10, 24], which suggested its potential as a novel target for anti-cancer therapy.

Although an important role for the KLF6 tumor suppressor gene in gastric cancer development and progression has been demonstrated [25], the role of KLF6-SV1 in gastric cancer remains largely unknown. Here, we found that KLF6-SV1 was overexpressed in gastric tumor samples, and in various gastric cancer cell lines. Moreover, in order to address the role of KLF6-SV1, we used RNA interference (RNAi) to produce specific and long-term silencing of KLF6-SV1 in the gastric cancer cell lines BGC-823 and SGC-7901. We evaluated the capacity for cell growth, migration, invasion, and survival of KLF6-SV1-silenced BGC-823 and SGC-7901 cell clones in vitro. Furthermore, the possible KLF6-SV1-downstream mediators and KLF6-
SV1-dependent signaling pathways were explored. Importantly, we used polyethylenimine as our gene delivery system (which overcame the limitations of the transience and high cost of synthetic siRNAs) to effectively inhibit BGC823 and SGC-7901 tumor growth in a nude mouse model.

\section{Materials and methods}

Tumor sample preparation

Human gastric cancer and adjacent non-cancer tissues were obtained from gastric cancer patients at the Tumor Hospital of Harbin Medical University during surgery. Matched non-cancer gastric samples were obtained at least $2 \mathrm{~cm}$ distant from the tumor, in which tumor cell infiltration was ruled out by histologic assessment. All specimens were snap-frozen and stored at $-80^{\circ} \mathrm{C}$. All patients gave their informed written consent for obtaining the specimens, and the study was approved by the Clinical Research Ethics Committee of the Harbin Medical University. The patient characteristics are shown in Table 1, and a total of 20 gastric tumor samples were analyzed.

siRNA sequence and construct

To silence KLF6-SV1 expression, we selected a candidate sequence in the KLF6-SV1 cDNA for RNAi, as described previously $[10,20,23,26]$. The targeted KLF6-SV1 sequence was $5^{\prime}$-CAGGGAAGGAGAAAAGCCUUU- $3^{\prime}$. The 21-nucleotide (nt) sequence showed no homology with other known human genes. Synthetic, annealed, siRNA oligonucleotides were synthesized chemically and

Table 1 Patient characteristics $(n=20)$

\begin{tabular}{ll}
\hline Characteristic & \\
\hline Median age, years (range) & $42(27-70)$ \\
Male/female ratio & $15 / 5$ \\
Tumor size (cm) & \\
$<5$ & $8(40 \%)$ \\
$\geq 5$ & $12(60 \%)$ \\
Clinical stage & \\
I & $2(10 \%)$ \\
II & $4(20 \%)$ \\
III & $6(30 \%)$ \\
IV & $8(40 \%)$ \\
Differentiation & \\
Well & $3(15 \%)$ \\
Moderate & $6(30 \%)$ \\
Poor & $9(45 \%)$ \\
Undifferentiated & $2(10 \%)$ \\
\hline
\end{tabular}


gel-electrophoresis purified (Ambion, Austin, TX, USA) and used during transient transfection experiments. Human mismatch or scrambled siRNA sequence (Ambion) possessing limited homology to human genes served as a negative control. For stable RNAi we designed a hairpin siRNA sequence that contains both sense and antisense siRNA sequences against KLF6-SV1. The annealed dsDNA oligonucleotides were ligated between the BamHI and HindIII sites on the pSilencer2.1-U6 hygro vector (Ambion). The control vector (si-ctrl) was constructed by inserting a sequence that expressed an siRNA with limited homology to sequences in the human genomes. All inserted sequences were verified by DNA sequencing.

\section{Cell culture and transfection}

The human gastric cancer cell lines MKN.28, SGC-7901, AGS, and BGC-823 were purchased from the Institute of Cell Biology (Shanghai, China). Cell lines were cultured in DMEM with $10 \%$ fetal bovine serum (FBS). Transfection was done with Superfect (Qiagen, Valencia, CA, USA) as directed by the manufacturer and selected in hygromycin $\mathrm{B}$ (Invitrogen Life Technologies, Carlsbad, CA, USA)-containing medium at $400 \mu \mathrm{g} / \mathrm{ml}$ for gastric cancer cells. Stable transfected cell clones were tested for KLF6-SV1 expression by reverse transcriptase polymerase chain reaction (RT-PCR) and Western blotting.

In vitro cell growth assay

The in vitro growth of siRNA-transfected cells was assessed at 24, 48, and $72 \mathrm{~h}$. Cells were grown in monolayer culture to $60 \%$ confluence, harvested using trypsin, and plated at a density of $5 \times 10^{3}$ cells/well into separate wells of a 96-well plate (Costar; Corning, Corning, NY, USA). DMEM-10\% FBS supplemented with $100 \mathrm{IU} / \mathrm{ml}$ penicillin and $100 \mathrm{mg} / \mathrm{ml}$ streptomycin was used as culture medium. The cells were incubated with 3-(4,5-Dimethylthiazol-2-yl)-2,5-diphenyltetrazolium bromide (MTT) $(0.5 \mathrm{mg} / \mathrm{ml})$ after 24,48 , and $72 \mathrm{~h}$. The color intensity was measured at $490 \mathrm{~nm}$ using an enzymelinked immunosorbent assay reader (Dynatech, Alexandria, VA, USA). The experiments were performed in triplicate. The cell viability was expressed absorbance as A490 nm.

For the anchorage-independent growth assay, cells were seeded in $0.3 \%$ agarose over a $0.6 \%$ agarose bottom layer at a density of 500 cells per well in a 24 -well plate. After 3 weeks, the numbers of colonies greater than $100 \mu \mathrm{m}$ in diameter were counted.

In vitro migration and invasion assays

The motility and invasiveness of plasmid-transfected cells were evaluated in 24-well Transwell chambers with upper and lower culture compartments separated by polycarbonate membranes with $8-\mu \mathrm{m}$ sized pores (Costar 3422; Corning). Prior to plating cells into the Transwells, DMEM- $0.1 \%$ bovine serum albumin (BSA) was incubated in the top chamber of each Transwell at $37^{\circ} \mathrm{C}$ for $1 \mathrm{~h}$ to saturate non-specific binding sites and then subsequently removed; $5 \times 10^{4}$ cells suspended in $100 \mu \mathrm{l}$ of DMEM$0.1 \%$ BSA were plated into the top chamber. DMEM-10\% FBS was placed into the bottom chamber to act as a chemoattractant. The cells that migrated through the $8-\mu \mathrm{m}-$ sized pores and adhered to the lower surface of the membrane were fixed with $3.7 \%$ paraformaldehyde, stained with $0.2 \%$ crystal violet, and washed with $1 \times$ phosphate-buffered saline (PBS) three times. The dye was eluted using $30 \%$ acetic acid, and quantification of cell number was performed using colorimetric analysis with a microplate reader (absorbance at $590 \mathrm{~nm}$ ). The absorbance units obtained for plasmid-transfected cells were each divided by the absorbance units obtained for untreated wild-type cells and expressed as a migration index. By definition, untreated wild-type cells were assigned an index of 1 .

In a similar fashion, the invasiveness of plasmid transfected cells was evaluated in Matrigel ${ }^{\mathrm{TM}}$ (Collaborative Biomedical Products, Bedford, MA, USA)-coated 24-well Transwell chambers. Matrigel was used at a concentration of $0.4 \mathrm{mg} / \mathrm{ml}$. Cells, media, experimental conditions, and analysis performed were similar to those for the migration assays. Triplicate assays were performed for each group of cells in both the migration and invasion assays, and results were expressed as means $\pm \mathrm{SD}$.

\section{Apoptosis analysis}

The apoptosis assay was conducted according to Fan et al. [27]. Briefly, gastric cancer cells were starved of serum for various time intervals and harvested by adding trypsinethylenediaminetetraacetate (EDTA) and resuspended in $1 \times$ binding buffer (BD PharMingen, San Diego, CA, USA) for terminal deoxynucleotidyl transferase dUTP nick end labeling (TUNEL) assay. A Fluorescein FragEL ${ }^{\mathrm{TM}}$ DNA Fragmentation Detection Kit (Calbiochem, Darmstadt, Germany) was used for the TUNEL assay. The fluorescence of cells carrying DNA labeled with fluorescein isothiocyanate (FITC) 2'-Deoxyuridine, 5'-Triphosphate (dUTP) (TUNEL-positive cells) was analyzed by BectonDickinson (BD, San Jose, CA, USA) FACscan. In addition, the entire cell cycle profile was analyzed by FACS with propidium iodide (PI) staining.

Caspase activity was assayed with a fluorometric kit purchased from R\&D Systems (R\&D Systems, Minneapolis, MN,USA). In brief, the cells were washed with icecold PBS and lysed in a cell lysis buffer provided in the kit. Then caspase-9 Leu-Glu-His-Asp-pNA (LEHD-pNA) or 
caspase-3 Asp-Glu-Val-Asp-pNA (DEVE-pNA) substrate was added to the cell lysates in a 96-well plate, and the plates were then incubated for $1 \mathrm{~h}$. The release of pNA was measured at $405 \mathrm{~nm}$ in a microplate reader.

Real-time quantitative RT-PCR analysis

Gene expression was quantified by real-time quantitative RT-PCR using QuantiTect SYRB Green dye (Qiagen, Valencia, CA, USA). DNA amplification was carried out using Icycler (Bio-Rad, Hercules, CA, USA) and the detection was performed by measuring the binding of the fluorescence dye SYBR Green I to double-stranded DNA. All primer sequences and methods for quantifying KLF6 and KLF6-SV1 were performed as previously described $[12,20,25,26]$. All experiments were done in triplicate and independently validated three times. All values were normalized to glyceraldehyde 3-phosphate dehydrogenase (GAPDH) levels.

\section{Western blot analysis}

Cell extracts for Western blotting were harvested in radioimmunoprecipitation assay buffer (Santa Cruz Biotechnology [Santa Cruz, CA, USA] standard protocol). Tumor tissue extracts were harvested and prepared in T-PER reagent (Pierce Biotechnology). Equal amounts of protein $(50 \mu \mathrm{g})$, as determined by a Bio-Rad detergent-compatible protein quantification assay, were loaded and separated by polyacrylamide gel electrophoresis (PAGE) and transferred to nitrocellulose membranes. Western blotting was performed using antibodies for KLF6-SV1 (Zymed, South San Francisco, CA, USA), KLF6 (SC-7158), and anti-plasminogen activation (uPA) (Santa Cruz Biotechnology, Santa Cruz, CA, USA), p-Akt and Akt (Boster Biotechnology, Wuhan, P. R. China), vascular endothelial growth factor (VEGF), Keratin 4, E-cadherin, matrix metalloproteinase (MMP)-9, caspase-3, poly ADP ribose polymerase (PRAP), Noxa, Mcl-1, Bim, Bak, Bax, and phosphatase and tensin homologue deleted on chromosome 10 (PTEN) (Beyotime, Haimen, P. R. China). GAPDH (Ambion) was used as an internal control. After probing with individual antibodies, antigen-antibody complex was visualized with the enhanced chemiluminescence reagent Supersignal (Pierce Biotechnology, Inc. Rockford, USA).

In vivo animal experiments

Six- to eight-week-old female nude mice were obtained from the Planned Parenthood Research Institute (Shanghai, P. R. China). All animals in this study were housed under pathogen-free conditions and were maintained in accordance with the guidelines of the Committee on Animals of the Second Military Medical University, Shanghai, China. Gastric cancer cells in mid-log-growth phase were harvested by trypsinization. Single-cell suspensions $\left(2 \times 10^{6}\right.$ cells in $0.1 \mathrm{~mL}$ Hank's buffered salt solution (HBSS)) were injected s.c. into the nude mice. The tumors were measured every 4 days with a caliper, and the diameters were recorded. Tumor volume was calculated by the formula: $a^{2} b / 2$, where $a$ and $b$ are the two maximum diameters. When tumors reached $2.0 \times 2.0 \mathrm{~cm}$, the duration of survival was recorded, and the mouse was euthanized.

For the KLF6-SV1 siRNA gene therapy experiment, $2 \times 10^{6}$ gastric cancer cells were injected s.c. into nude mice. Seven days later, when the tumors were palpable, the mice were divided into three groups. Group 1 mice were used as untreated controls (PBS treatment). Group 2 received intratumoral injection $(20 \mu \mathrm{g} /$ mouse $)$ of polyethylenimine/control (PEI/si-ctrl) vector on day 7. Group 3 received intratumoral injection with $20 \mu \mathrm{g}$ polyethylenimine/si-SV1 (PEI/si-SV1). Tumor size was measured as described above.

Statistical analysis

Data are expressed as means \pm SD. Statistical analysis of data was done with Student's $t$-test, using SigmaPlot software (St. Louis, MO, USA). Differences were considered to be statistically significant at $P<0.05$.

\section{Results}

Expression of KLF6 and KLF6 alternative splicing, KLF6-SV1 in gastric tumors and cell lines

Previous studies have demonstrated decreased expression of KLF6 in several tumors, such as non-small cell lung cancer [9], colorectal cancer [7], and ovarian cancer [20]. Here, real-time PCR analysis using KLF6-specific primers was performed in samples from patients with primary gastric cancer. Compared with control normal tissue, all gastric cancer samples showed a significant reduction in KLF6 expression, which was consistent with previous reports $[11,25]$. However, the expression of KLF6-SV1 was increased in samples from the primary gastric cancer patients, compared with control normal tissue (Fig. 1a).

Furthermore, four well-characterized gastric cancer cell lines, MKN.28, SGC-7901, AGS, and BGC-823, were chosen to determine KLF6-SV1 expression, due to their diverse differentiation features. The levels of wild-type KLF6 (wtKLF6) and KLF-6-SV1 in these cell lines were determined and compared at both the mRNA and protein levels. As shown in Fig. 1b, c, the mRNA level of KLF6SV1 was higher in the undifferentiated cell line BGC-823 

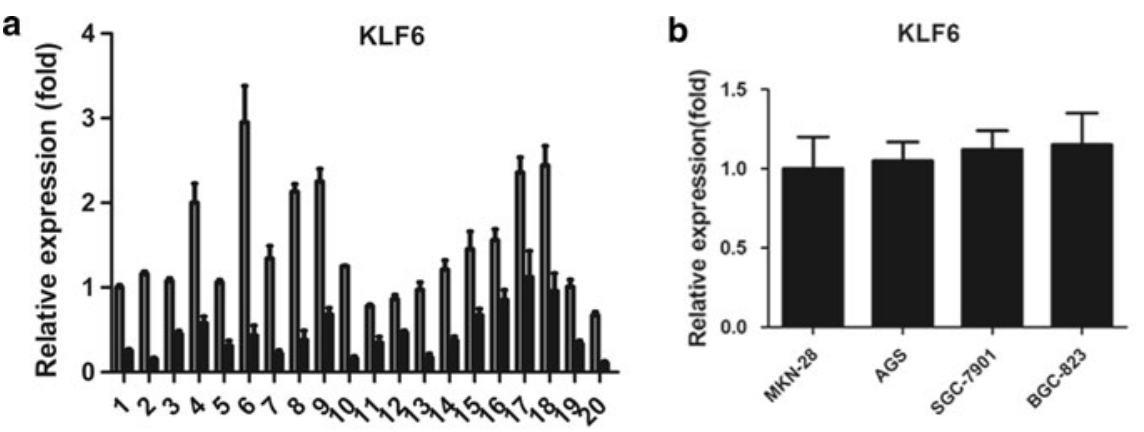

KLF6-SV1
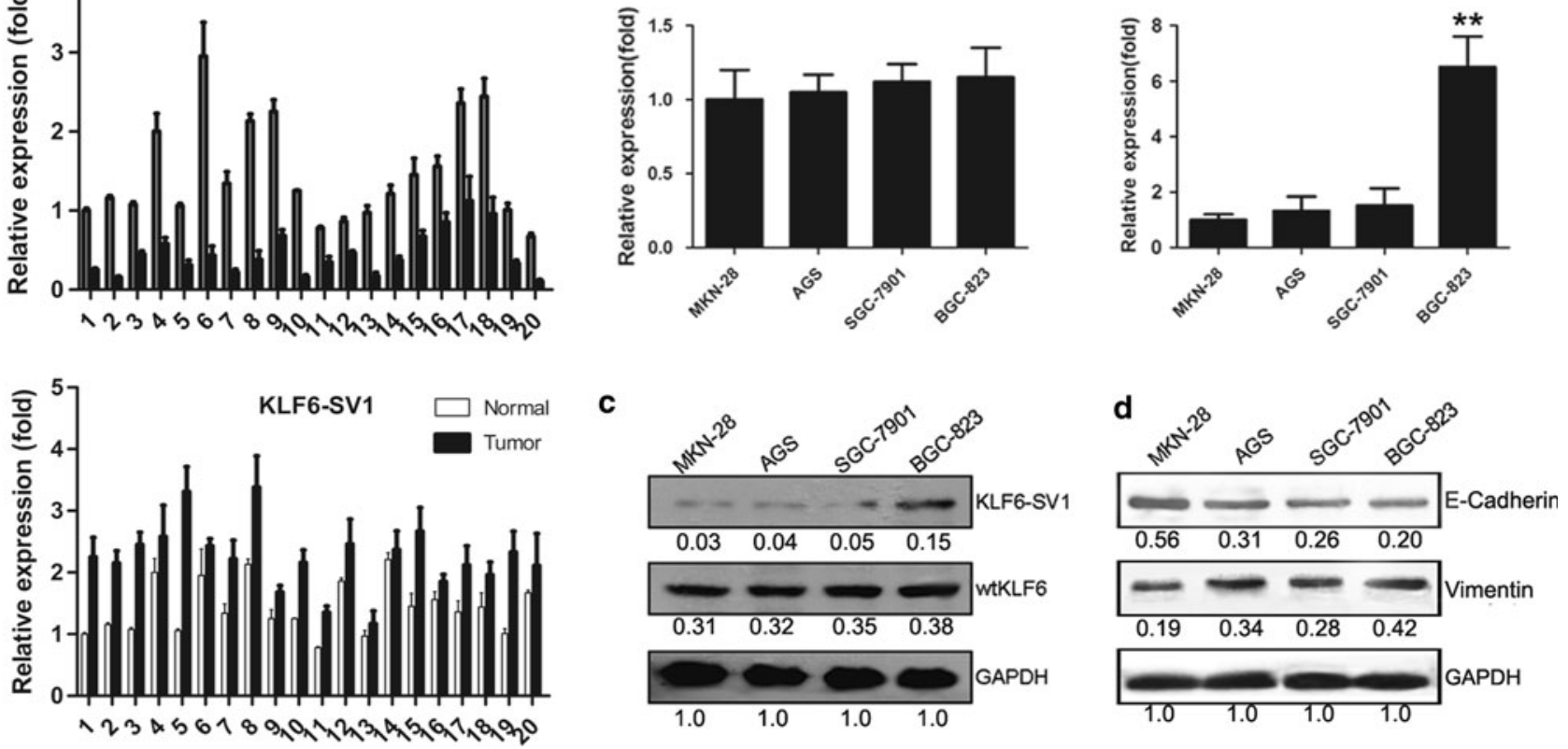

Fig. 1 Expression of KLF6 and KLF6-SV1 in samples from gastric cancer patients and cell lines. a mRNA expression of KLF6 and KLF6-SV1 in each tumor/normal pair. b mRNA expression of KLF6 and KLF6-SV1 in various gastric cancer cell lines. c Western blotting

analysis for KLF6 and KLF6-SV1 expression in gastric cancer cell lines. The glyceraldehyde 3-phosphate dehydrogenase (GAPDH) level served as an internal control. $* * P<0.01$ compared with other groups

than that in MKN.28, AGS, and SGC-7901 cells, whereas the wtKLF6 level showed no difference among these cell lines (Fig. 1b). Furthermore, Western-blotting analysis of KLF6 and KLF6-SV1 showed similar results in these cell lines. An average 2.5-fold increase in the ratio of KLF6SV1 to wtKLF6 was observed in BGC-823 cells, compared with the other cell lines (Fig. 1c). In addition, we found that the trend of vimentin expression in the four cell lines was consistent with that of KLF6-SV1, and E-cadherin expression was inversely correlated with that of KLF6SV1.

RNAi decreases KLF6-SV1 expression in gastric cancer cells

Having shown differential regulation of the KLF6-SV1 level in these four cell lines, and to investigate the role of KLF6-SV1 in the development of gastric tumors, we used RNAi to suppress KLF6-SV1 expression in the BGC-823 and SGC-7901 cell lines. As shown in Fig. 2a, KLF6-SV1 expression was significantly inhibited by si-SV1 transient transfection. In si-SV1 transient-transfected cells, the KLF6-SV1 level was reduced by $\sim 65$ and $\sim 85 \%$, at 24 and $48 \mathrm{~h}$, respectively, compared with WT BGC-823. Similarly, si-SV1 transient transfection also reduced the expression of KLF6-SV1 in the SGC-7901 cell line. No significant difference was observed in KLF6-SV1 protein levels between WT and si-ctrl transfected cells (Fig. 2a, b). This RNAi silencing effect was specific, as the GAPDH level did not differ significantly among the treated cells and controls. In addition, we found that the expression of wtKLF6 was not affected by KLF6-SV1 siRNA (data not shown).

In order to assess the features of tumor cells in which KLF6-SV1 expression was inhibited over the long-term, we directed our attention to generating stable, KLF6-SV1 downregulated clonal cell lines. BGC-823 and SGC-7901 cell lines were transfected with purified pSilencer2.1-U6 hygro vector containing either the SV1-siRNA insert (si-SV1) or the control siRNA insert (si-ctrl). One month after stable transfection and screening, RT-PCR and Western blotting analysis of three clones in the BGC-823 cell line demonstrated decreased KLF6-SV1 mRNA and protein expression, compared with si-ctrl cells. Among them, the BGC/si-SV1-3 clone showed the most significant decrease in KLF6-SV1 expression. Similarly, in the SGC7901 cell line, three clones with stable transfection also showed significantly reduced RNA and protein expression of KLF6-SV1. Among them, the expression of KLF6-SV1 in clone SGC/si-SV1-1 was almost completely suppressed (Fig. 2c, d). Moreover,the mRNA and protein levels of wtKLF6 were not affected by KLF6-SV1 siRNA in the stable transfected cell lines (data not shown), indicating that the RNAi-mediated downregulation of KLF6-SV1 was 
Fig. 2 Inhibition of KLF6-SV1 expression in BGC-823 and SGC-7901 cell lines by RNA interference (RNAi). a, b Western blotting showing that the RNAi-mediated silencing of KLF6-SV1 by transient transfection is time-dependent. The extent of KLF6-SV1 suppression in the gastric cancer cell lines BGC-823 (a) and SGC-7901 (b) was measured at 24 and 48 h. c, d Suppression of KLF6-SV1 mRNA and protein in BGC-823 (c) and SGC-7901 (d) stably transfected with small interfering (si)-SV1 at 1 month after initial transfection. Upper panels mRNA data, bottom panels Western blotting data. $* * P<0.01$ compared with control group. WT wild type a

BGC-823
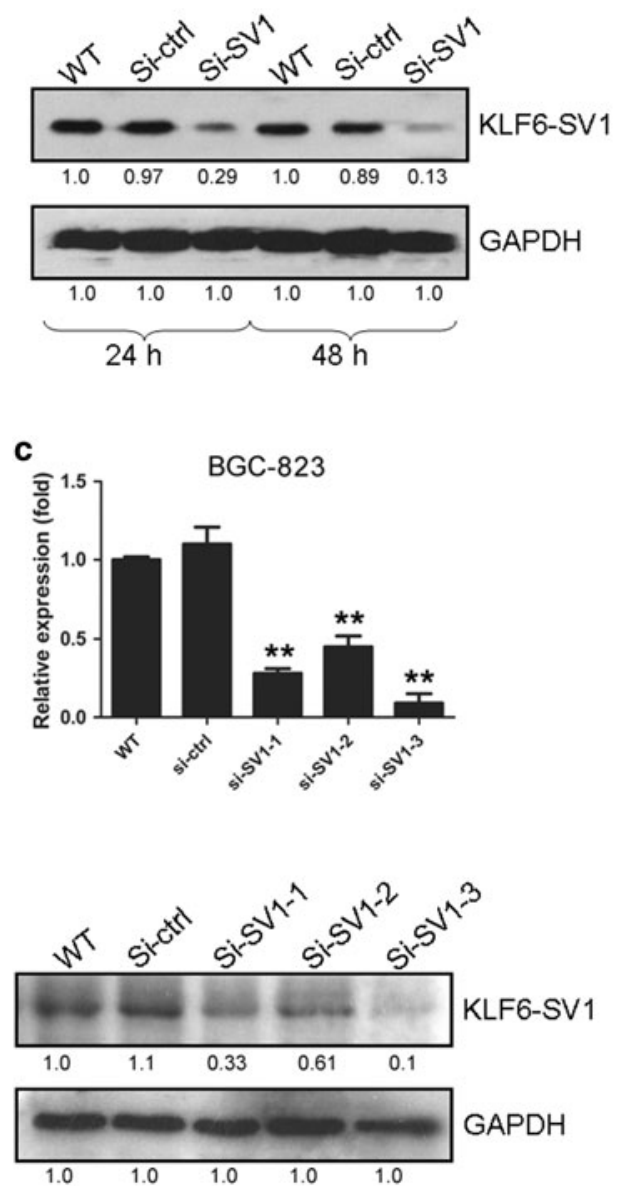

b

SGC-7901
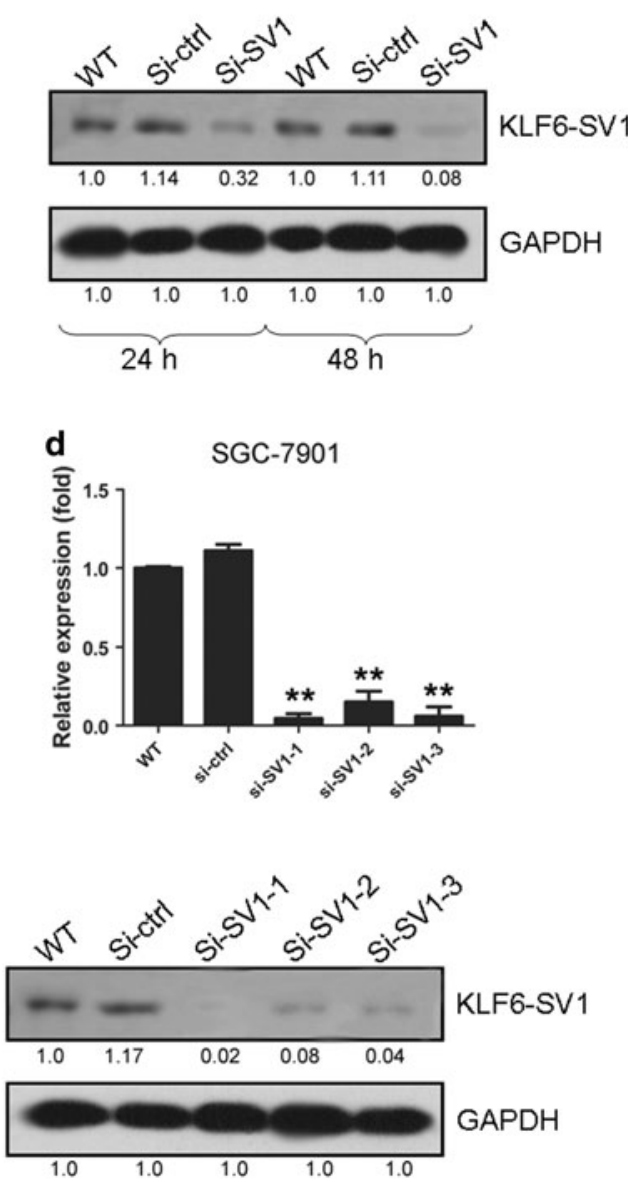

specific and did not result in a global decrease in gene expression.

KLF6-SV1 siRNA suppresses gastric cancer cell growth, colony formation, migration, and invasion

It has been reported that overexpression of KLF6-SV1 could enhance cell growth, migration, and invasion in several kinds of tumor cells [10, 13, 20-23]. Thus, to examine the effect of the targeted downregulation of KLF6-SV1 specifically by RNAi-mediated gene silencing on the growth and metastasis-related features of gastric cancer cells, we selected the siRNA-SV1-transfected clone in which the expression of KLF6-SV1 was mostly inhibited in the BGC-823 and SGC-7901 cell lines. We first investigated the effect of KLF6-SV1 siRNA on gastric cancer cell growth and anchorage-independent growth in vitro. As shown in Fig. 3a, b, KLF6-SV1 siRNA, but not control siRNA, significantly inhibited the growth and anchorageindependent growth of BGC-823 and SGC-7901 cells. Similar rates of growth were observed between WT and sictrl transfected cells.
We further evaluated whether the suppression of KLF6SV1 expression could alter the ability of the in vitro migration of BGC-823 and SGC-7901 cells. As shown in Fig. 3c, cell motility in BGC/si-SV1-3 and SGC/si-SV1-1 was reduced by 25 and $21 \%$, respectively, compared with WT and si-ctrl cells. We also determined whether KLF6SV1 silencing affected the ability of gastric cancer cells to invade into Matrigel ${ }^{\mathrm{TM}}$. As shown in Fig. 3d, BGC/si-SV13 and SGC/si-SV1-1 showed 85 and $90 \%$ decreased invasiveness, respectively, compared with WT and si-ctrl cells. Taken together, these data suggest that the downregulation of KLF6-SV1 expression by siRNA resulted in decreased proliferation, cell motility, and invasiveness of gastric cancer cells in vitro.

KLF6-SV1 siRNA enhances the apoptosis of gastric cancer cells

Tumor cells are often deficient in apoptotic regulation, which leads to the expansion of tumor cells, metastasis, and invasion [28, 29]. It was demonstrated that KLF6-SV1 was an important anti-apoptosis protein in some tumors [26]. 

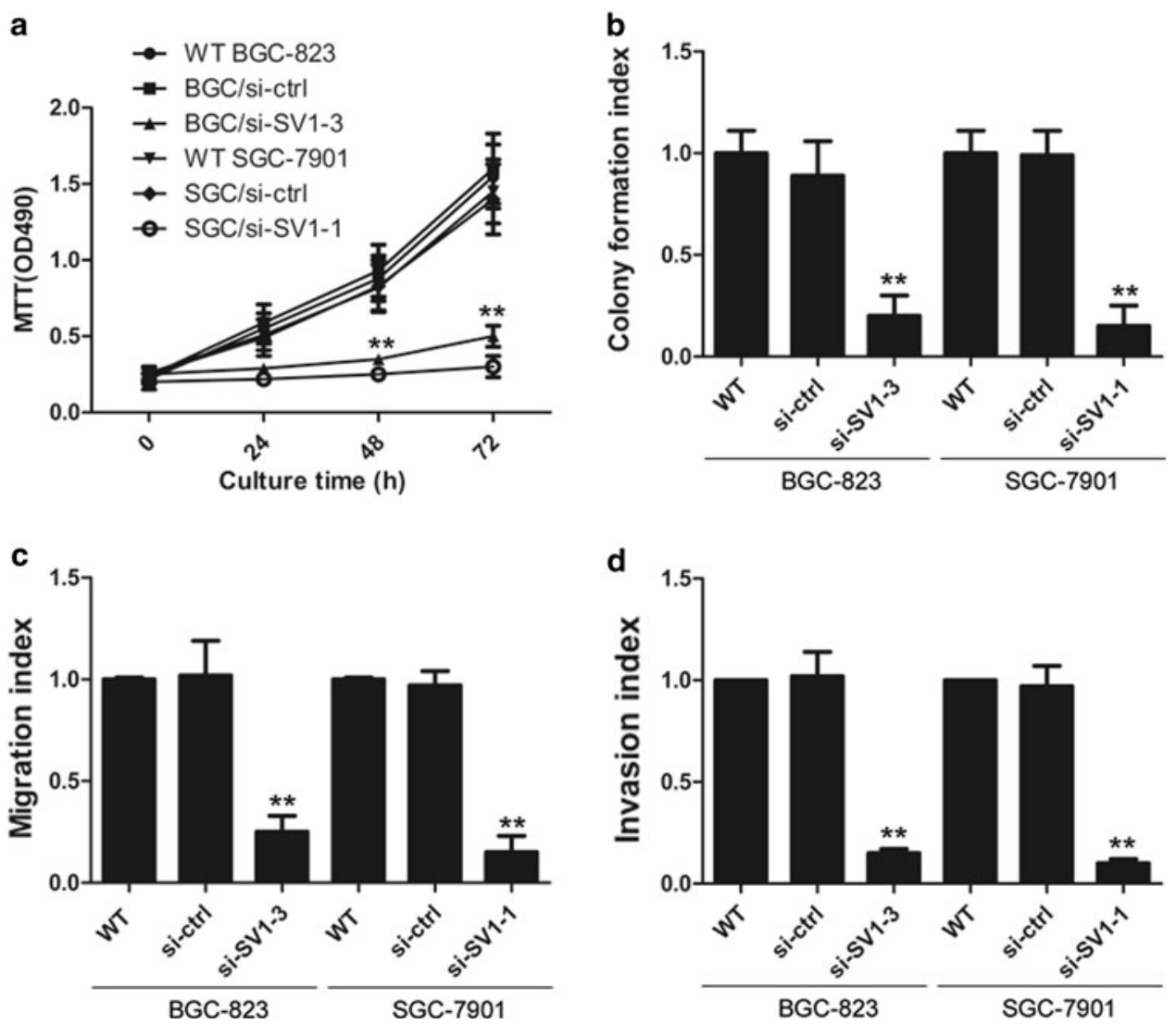

Fig. 3 Effect of KLF6-SV1 siRNA on gastric cancer cell growth, migration, and invasiveness. a Effect of KLF6-SV1 siRNA on gastric cancer cell proliferation. Stably transfected gastric cancer cell lines were cultured in 96-well plates for various times. Then cell proliferation was determined by MTT assay. Values are given as the means \pm SD of 6 wells. b Effect of KLF6-SV1 siRNA on anchorage-independent growth of gastric cancer cells. Stably transfected gastric cancer cells were seeded in semisolid soft agar medium to monitor anchorage-independent growth. c Effect of KLF-SV1 siRNA on gastric cancer cell migration. The migration was assessed

Therefore, we investigated the effect of KLF6-SV1 downregulation on the survival of gastric cancer cell lines in vitro. Under starvation conditions, transfection with KLF6-SV1 siRNA dramatically increased the percentage of TUNEL-positive apoptotic cells in BGC/si-SV1-3 and SGC/si-SV1-1 clone cells compared to the controls (Fig. 4a). The broad-spectrum caspase inhibitor benzyloxycarbonyl-Val-Ala-Asp (OMe) fluoromethylketone (zVAD-fmk) significantly inhibited KLF6-SV1 siRNAinduced cell death (Fig. 4a), indicating that the downregulation of KLF6-SV1-induced apoptosis was caspase-dependent in both the gastric cancer cell lines. Furthermore, the data of the entire cell-cycle analysis also demonstrated the role of KLF6-SV1 siRNA. In addition, we examined the levels of caspase-3 and -9 , the critical parameters for apoptosis. The activities of caspase- 3 and -9 were also increased in KLF6-SV1 siRNA-transfected cells (Fig. 4b). The cleaved PRAP and active caspase- 3 levels in both of

by incubating cells in Transwell chambers for $24 \mathrm{~h}$. Migrated cells were stained with $0.2 \%$ crystal violet, subjected to elution with acetic acid, and quantified in a microplate reader (A590 nm). By definition, WT cells were assigned a migration index of 1. d Effect of KLF-SV1 silencing on gastric cancer cell invasiveness. The invasiveness was assessed by incubating cells in Matrigel-coated Transwell chambers for $24 \mathrm{~h}$. WT cells were assigned an invasion index of 1 . The data represent the mean $\pm \mathrm{SD}$ of triplicate assays. $* * P<0.01$ compared with control group

the gastric cancer cell lines indicated by Western blotting showed significant upregulation after KLF6-SV1 siRNA transfection.

KLF6-SV1 siRNA alters the expression levels of tumorigenicity related-genes

Ki-67 antigen is commonly used as a marker of cell proliferative activity. Therefore, we determined the changes of Ki-67 expression in BGC-823 and SGC-7901 gastric cancer cells after KLF6-SV1 siRNA transfection. As shown in Fig. 5, the protein and mRNA expression of Ki-67 was significantly reduced in BGC/si-SV1-3 and SGC/si-SV1-1 clone cells, compared to the controls.

It has been shown that several proteins associated with KLF6-SV1 function, such as MMP-9, Keratin 4, E-cadherin, VEGF, and uPA play important roles in tumor growth, invasion, and angiogenesis [30]. Therefore, we 
Fig. 4 Effect of KLF-SV1 siRNA on gastric cancer cell survival. a Stably transfected gastric cancer cells were cultured and exposed to serum starvation for various times. Then apoptotic cells were detected by flow cytometry with TUNEL staining. In some experiments, the caspase inhibitor zVAD-fmk $(80 \mu \mathrm{M})$ was added to the test. b Effect of KLF-SV1 siRNA on gastric cancer cell cycle. The entire cell cycle profile was analyzed by FACS with PI staining. c Effect of KLF-SV1 siRNA on caspase3 or caspase- 9 activity. Cell lysates from stably transfected gastric cancer cells were incubated with the caspase- 3 or caspase- 9 substrate. The release of p-nitroanilide (pNA) was measured by absorbance at $405 \mathrm{~nm}$. Values are given as the mean \pm SD of 6 wells. $d$ The levels of cleaved PARP and active caspase 3 were determined by Western blotting. $* * P<0.01$ compared with control group
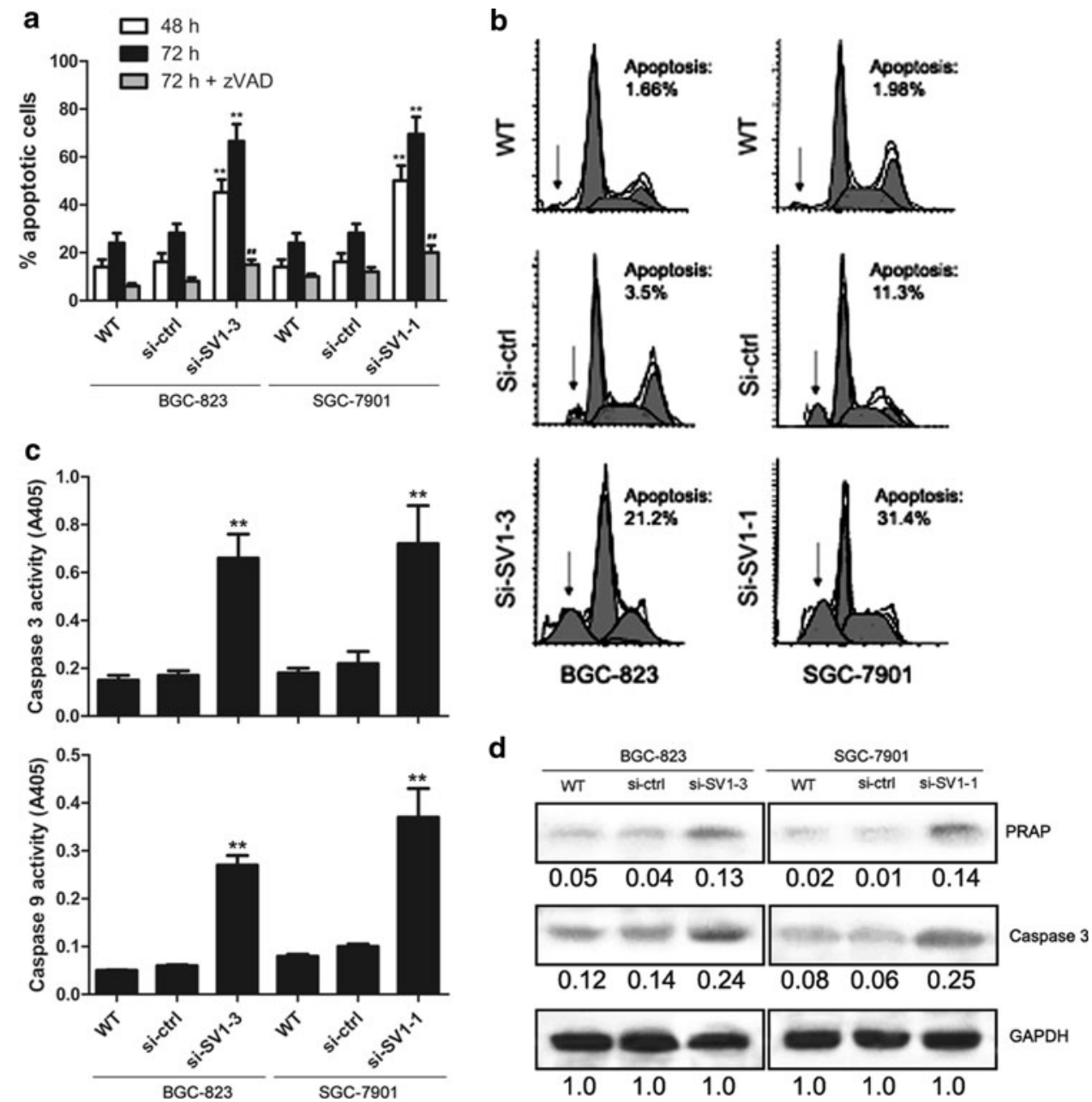

a

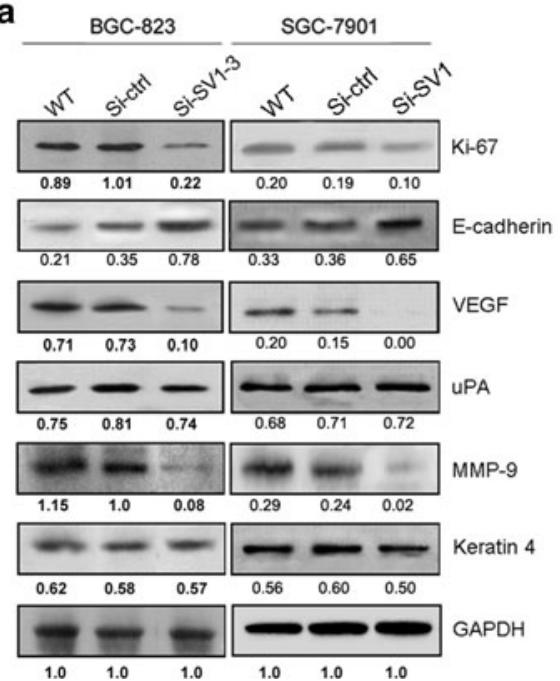

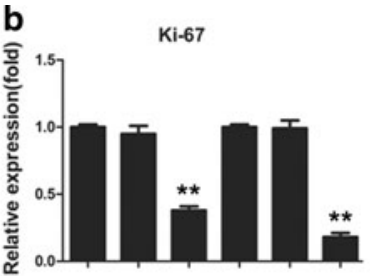

UPA

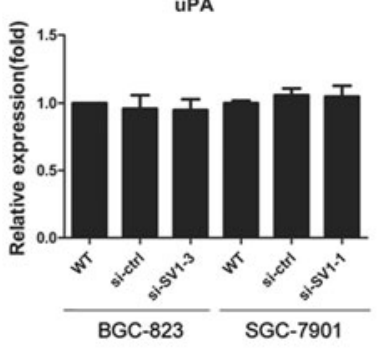

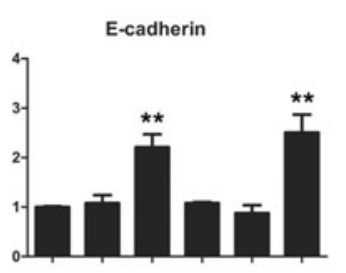

MMP-9

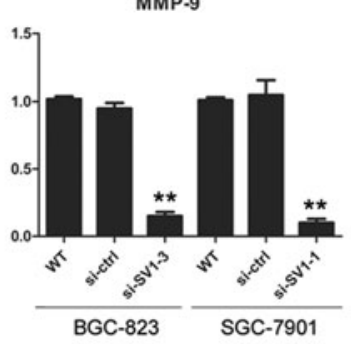

VEGF

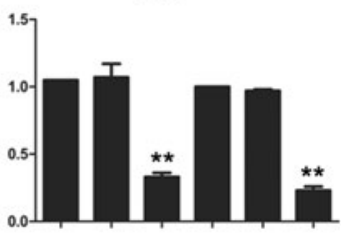

Keratin 4

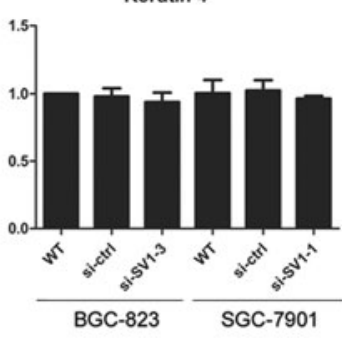

Fig. 5 KLF6-SV1 siRNA suppressed Ki-67, E-cadherin, vascular endothelial growth factor (VEGF), and matrix metalloproteinase (MMP)-9 protein and mRNA expression in gastric cancer cells. a Protein expressions associated with migration, proliferation, and invasion in WT, KLF6-SV1 downregulated clones (si-SV1-1 to siSV1-3), and control siRNA clonal cell lines were determined by
Western blotting. Western blotting with antibodies specific for Ki-67, Keratin 4, E-cadherin, MMP-9, uPA, and VEGF is shown. AntiGAPDH was used as an internal control. b mRNA expressions of these genes were determined by real-time reverse transcription polymerase chain (RT-PCR). $* * P<0.01$ compared with control group 
checked whether KLF6-SV1 siRNA affected the expression of these proteins in gastric cancer cells. The protein levels of MMP-9 and VEGF were decreased in both of the KLF6-SV1-downregulated clones of gastric cancer cell lines. In contrast, E-cadherin expression showed upregulation after KLF6-SV1 siRNA transfection. No significant differences were detected for Keratin 4 and uPA expression between the treatment and control groups (Fig. 5a). Furthermore, we found that the mRNA changes of these genes were consistent with the observed protein changes (Fig. 5b). Thus, these data indicated that the effect of KLF6-SV1 siRNA on tumor suppression might be mediated through the altered expression of MMP-9, E-cadherin, and VEGF.

KLF6-SV1 siRNA regulates apoptosis signaling in gastric cancer cells

Previous reports have demonstrated that the targeted reduction of KLF6-SV1 resulted in marked activation of the intrinsic pathway of apoptosis through upregulation of the pro-apoptotic gene Noxa and degradation of the antiapoptotic gene Mcl-1 [10, 24, 26]. To determine whether the KLF6-SV1 siRNA-induced apoptosis in gastric cancer cells was mediated by Noxa and Mcl-1, we analyzed the expression of both Noxa and Mcl-1 at the protein level. As shown in Fig. 6, KLF6-SV1 siRNA-transfected BGC-823 and SGC-7901 gastric cancer cells showed a marked increase in Noxa expression, with a concomitant decrease in the expression of Mcl-1. In addition, other proteins designated 'Bcl-2 family proteins', including Bim, Bak, and Bax, which are also critical in cell survival, were further determined [31-33]. As shown in Fig. 6, KLF6SV1-silencing resulted in an obvious decrease of Bim, and a significant increase of Bak expression. However, Bax expression was unchanged after KLF6-SV1 siRNA transfection. Therefore, it seems that the downregulation of KLF6-SV1-induced cell apoptosis is probably regulated by the quantitative balance between proapoptotic and antiapoptotic Bcl-2 family proteins.

Accumulated evidence has demonstrated that the activity of phosphatidylinositol 3-OH kinase (PI3K) and the phosphorylation of the serine/threonine kinase Akt (also called 'protein kinase B') play important roles in tumor cell survival [34]. Therefore, we determined whether KLF6SV1 could affect the activation of PI3K/Akt in gastric cancer cells. As shown in Fig. 6, we found that the levels of Akt phosphorylation and PDK1, which could activate Akt, were effectively suppressed in KLF6-SV1 siRNA-transfected cells. The level PTEN, a negative regulator of PI3K/ Akt signaling, was not affected by KLF6-SV1 silencing. Thus, the PI3K/Akt pathway was involved in the induction of apoptosis by KLF6-SV1 siRNA.

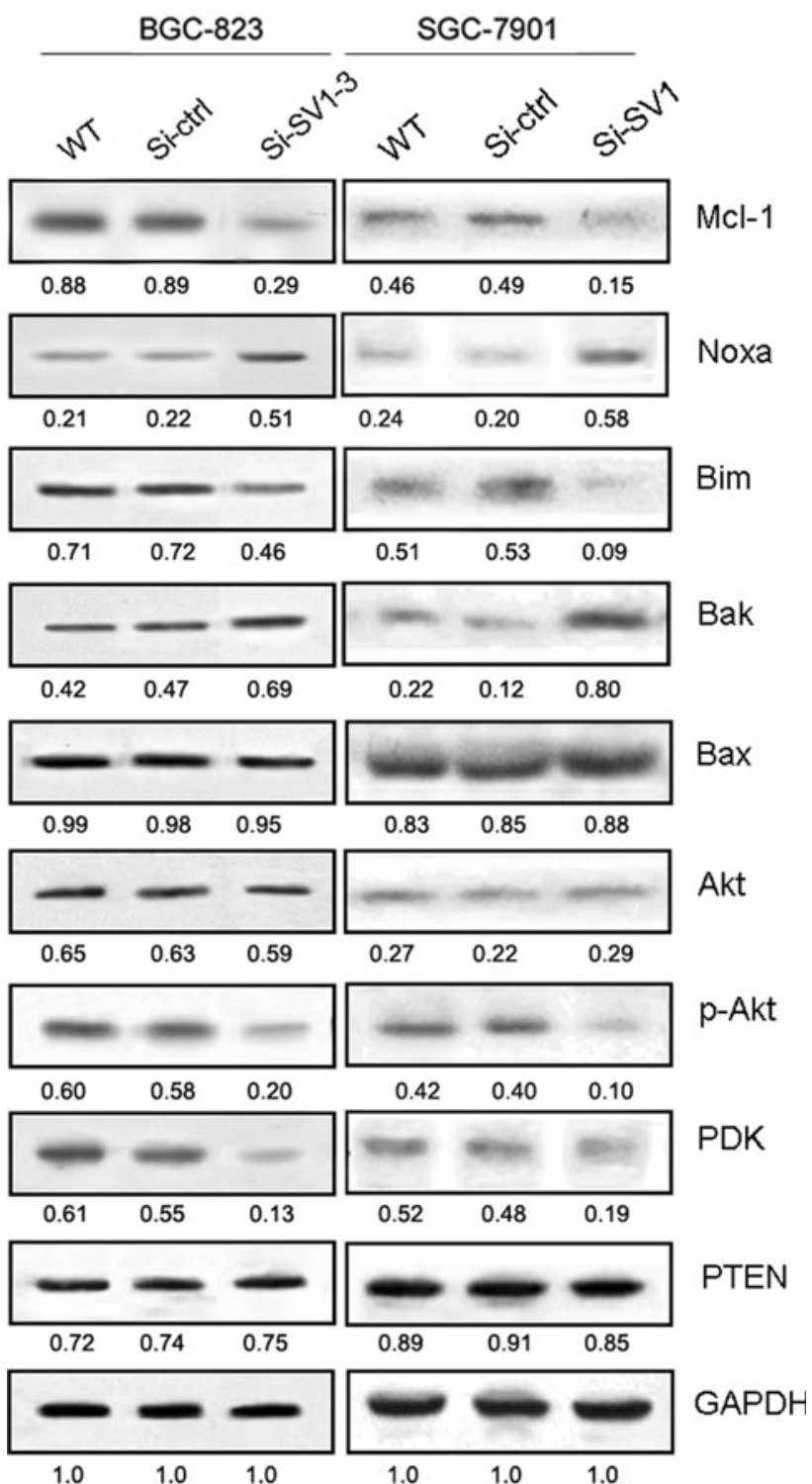

Fig. 6 KLF6-SV1 siRNA regulated the apoptosis signaling in gastric cancer cells. Cytoplasmic protein extracts were harvested and subjected to Western blotting with anti-Mcl-1, anti-Noxa, anti-Akt, anti-p-Akt, anti-PTEN, anti-PDK1, anti-Bak, anti-Bax, and anti-Bim. Anti-GAPDH was used as an internal control. Representative Western blot is shown

Effect of KLF6-SV1 siRNA on tumorigenicity of gastric cancer cell lines in vivo

To determine whether the inhibition of KLF6-SV1 expression by siRNA affected tumorigenicity in vivo, gastric cancer cell lines were inoculated s.c. into nude mice. As shown in Fig. 7a, WT and si-ctrl gastric cancer cells grew rapidly, resulting in palpable tumors 6-7 days following inoculation (Fig. 7a). By contrast, in BGC/siSV1-3 and SGC/si-SV1-1 clone cell groups, tumor formation was significantly slower, and the tumor size was 

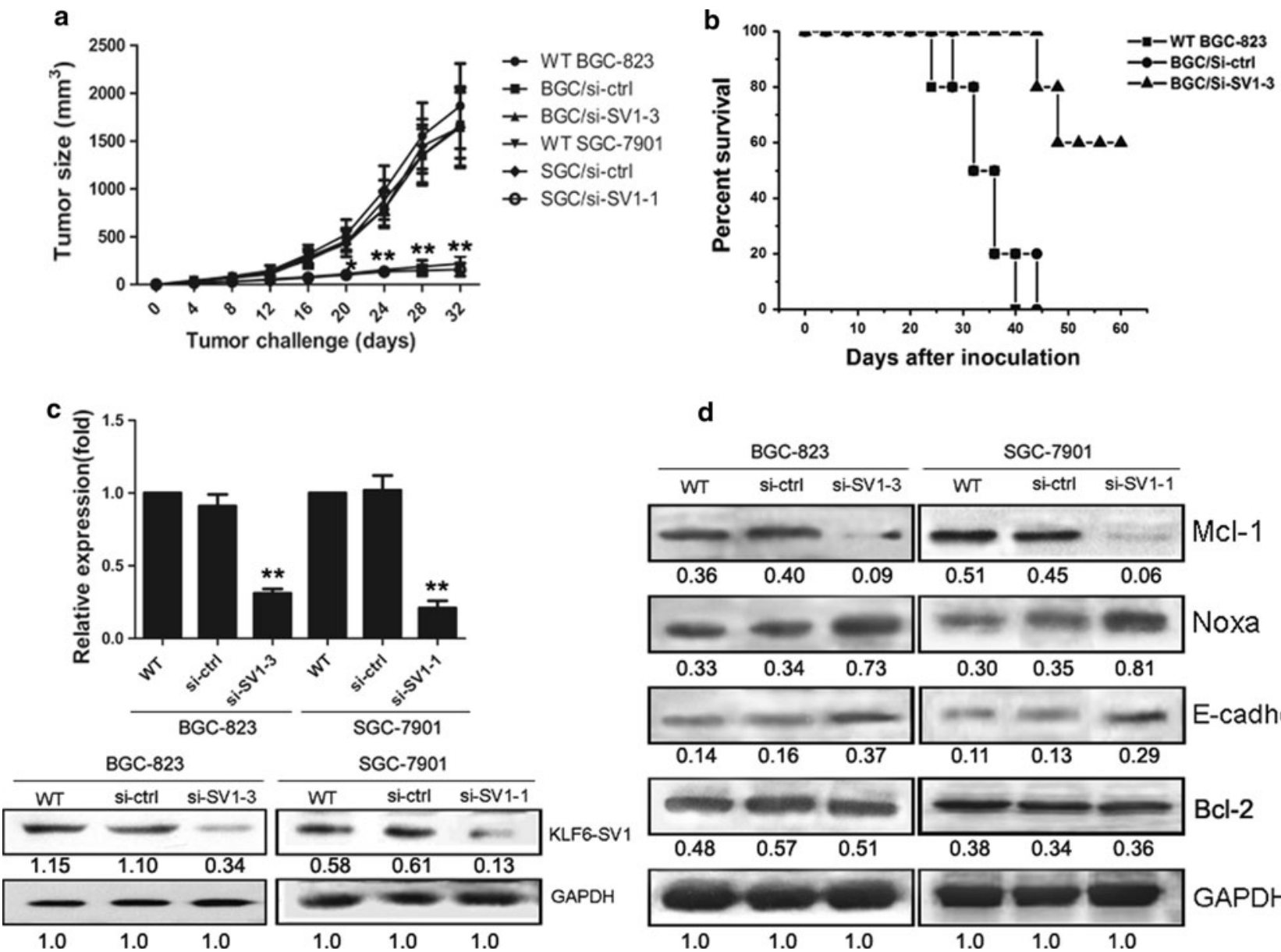

Fig. 7 Effect of KLF6-SV1 siRNA on xenograft gastric tumor growth. a KLF6-SV1 siRNA-transfected gastric cancer cells were inoculated s.c. into nude mice. Tumor growth was monitored and tumor volume was calculated ( $n=10$ each group). b Mice were euthanized when the tumor reached $2 \times 2 \mathrm{~cm}$ in diameter. The survival curve was calculated as the percentage of surviving mice on

remarkably smaller than the sizes in both control groups. Moreover, the survival time of tumor-bearing mice in the si-SV1-transfected group was significantly prolonged compared with the control groups (Fig. 7b). No significant difference in either tumor growth or survival time was observed between the two groups of si-ctrl and WT gastric cancer cells.

We further determined whether the downregulation of KLF6-SV1 expression was maintained in vivo by performing quantitative real-time PCR and Western blotting at the end of the experiment. As predicted, tumors derived from both of the si-SV1-transfected groups showed significantly decreased KLF6-SV1 levels compared with si-ctrl tumors (Fig. 7c), indicating that the inhibition of KLF6-SV1 expression by siRNA was effective and stable in vivo. Moreover, Mcl-1, Noxa, and E-cadherin also showed decreased expression in the si-SV1-transfected d

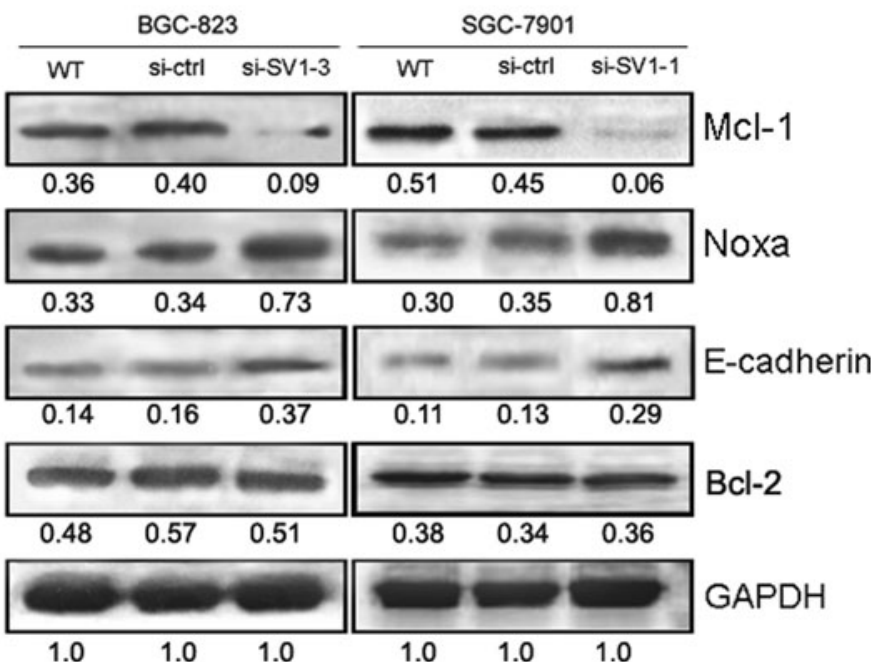

the indicated days. $\mathbf{c}$ mRNA and protein levels of KLF6-SV1 in tumor tissue were determined by quantitative real-time PCR and Western blotting. d The levels of Noxa, E-cadherin, Mcl-1, and Bcl-2 in the tumor xenografts were determined by Western blotting. $* * P<0.01$ compared with WT gastric tumors

groups, which was consistent with the in vitro observations. However, the Bcl-2 level in the si-SV1-transfected groups was unchanged.

Effect of KLF6-SV1 siRNA gene therapy on gastric tumor growth in vivo

To further investigate whether KLF6-SV1 siRNA could be used as a gene therapy for tumors, we selected polyethylenimine (PEI) as our gene delivery system because of a previous successful report with this nonviral vector [35]. As shown in Fig. 8a, PEI/si-SV1 intratumoral injection significantly inhibited tumor growth in mice, compared with the PEI/si-ctrl treatment. Moreover, we observed consistent results in the BGC-823 and SGC-7901 gastric cancer cell lines. Survival time for the PEI/si-SV1-treated mice was also significantly prolonged, compared with the 

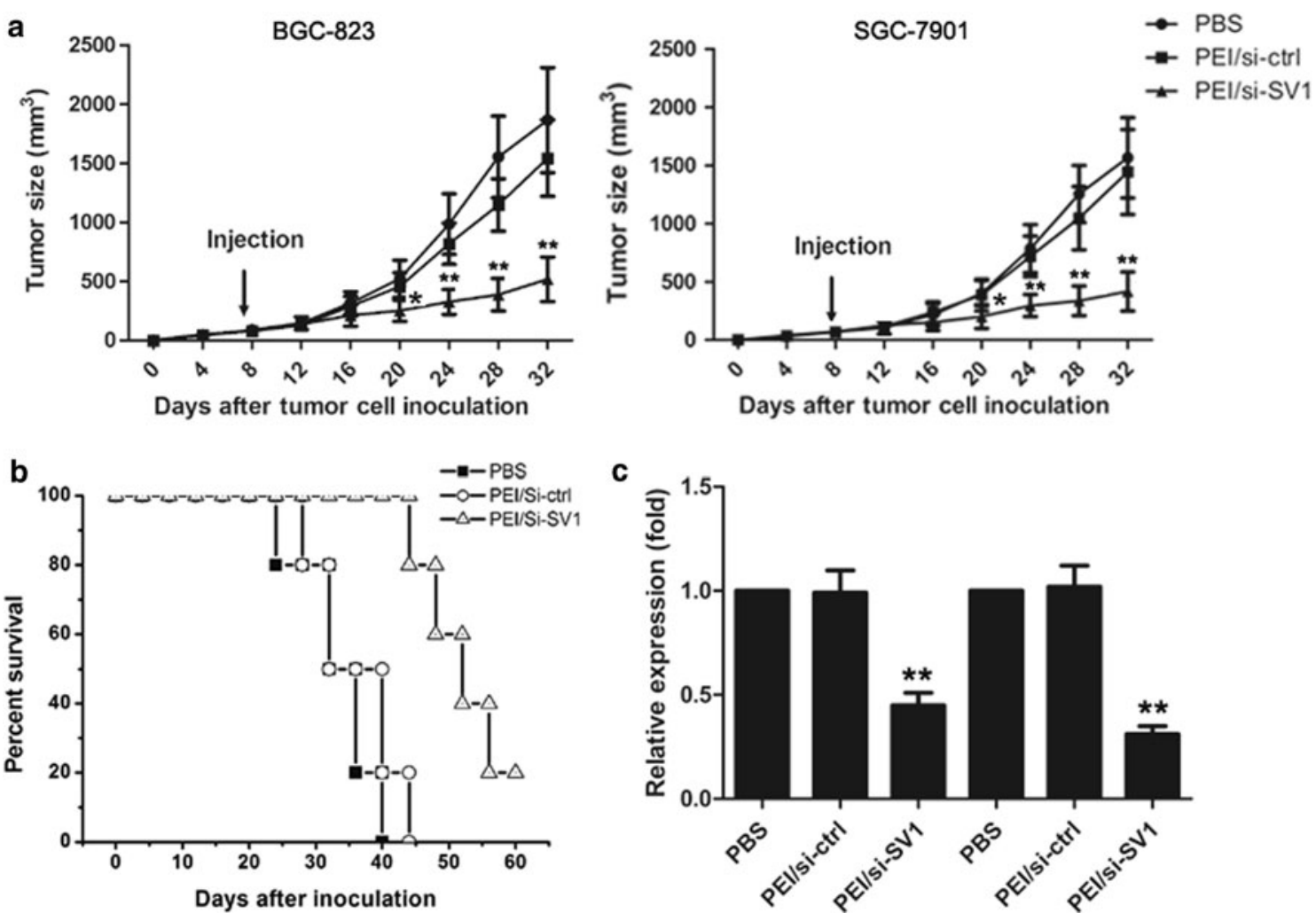

C

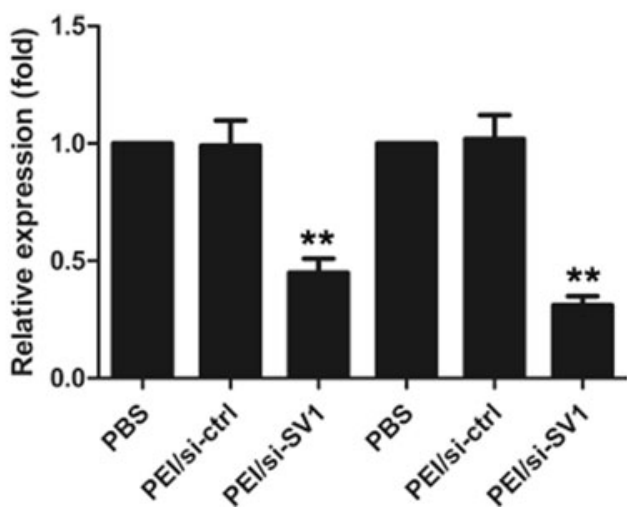

Fig. 8 Effect of KLF-SV1 siRNA gene therapy on gastric tumor growth in nude mice. a Gastric cancer cells were injected subcutaneously into nude mice. Seven days later, mice were divided into three groups and given intratumoral injections of polyethylenimine/sictrl, polyethylenimine/si-SV1, or phosphate-buffered saline $(P B S)$ treatment ( $n=10$ each group). Tumor size was measured at various

control groups (Fig. 8b). In addition, we also found that KLF6-SV1 mRNA levels in the PEI/si-ctrl-treated groups were significantly decreased. These results indicate that PEI used as an SV1 RNAi delivery system could effectively reduce KLF6-SV1 expression, and inhibit gastric tumor growth and improve animal survival in a nude mouse model.

\section{Discussion}

In the present study, we found increased expression of KLF6-SV1 in gastric cancer samples, indicating a role of KLF6-SV1 in the development of gastric cancer. Moreover, the level of KLF6-SV1 expression might be closely related to gastric tumor stage and grade, as indicated by the differential expression of KLF6-SV1 in diverse differentiated gastric cancer cell lines. It has been demonstrated that KLF6-SV1 overexpression resulted in marked changes in 3 key pathways regulating tumor growth and dissemination: apoptosis, cellular proliferation, and angiogenesis. Each time points. $* P<0.05$ compared with PBS-treated group. b Mice were euthanized when the tumor reached $2 \times 2 \mathrm{~cm}$ in diameter. The survival curve was calculated as the percentage of surviving mice on the indicated days. $\mathbf{c}$ mRNA level of KLF6-SV1 in tumor tissue was determined by quantitative real-time PCR. $* * P<0.01$ compared with control treatment

pathway is believed to play a critical role in tumor growth and metastasis. Therefore, inhibition of KLF6-SV1 theoretically represents an intriguing therapeutic target, because multiple pathways would be inhibited simultaneously [30]. Here, for the first time, we have reported that gene therapy targeting KLF6-SV1 might have a therapeutic benefit for gastric tumors. The gene therapy was performed by selectively inhibiting KLF6-SV1 expression using siRNA. After treatment with siRNA, the expression of KLF6$\mathrm{SV} 1$ protein was greatly reduced in the gastric cancer cell lines BGC-823 and SGC-7901. We investigated the effects of KLF6-SV1 siRNA on the proliferation, migration, invasion, and survival of both gastric cancer cell lines in vitro, and tumor growth in vivo.

In the present study, our data showed that KLF6-SV1 silencing effectively suppressed the proliferation, migration, and invasion of the gastric cancer cell lines, BGC-823 and SGC-7901, a finding that has not been reported previously. It is well known that KLF6-SV1 can regulate a repertoire of cancer related-genes, including Keratin 4, E-cadherin, MMP-9, uPA, and VEGF, which mediate 
angiogenesis, motility, and cell proliferation/survival [30, 31]. Here, we found that RNAi targeting KLF6-SV1 not only downregulated KLF6-SV1 expression, but also reduced KLF6-SV1 downstream targets and mediators, including MMP-9 and VEGF expression, and upregulated E-cadherin expression. Owing to the altered expression of these proteins, KLF6-SV1 siRNA-transfected gastric cancer cells were impaired in motility and invasion, and slower in tumor growth in vitro. In addition, KLF6-SV1 could antagonize the tumor suppressor function of KLF6, which has been associated with key pathways that are disrupted in human cancer, including p53-independent upregulation of p21 [5], reduced interaction of cyclin D1 with CDK4 [36], and the inhibition of c-jun [37]. Thus, we cannot exclude the possibility that KLF6-SV1 siRNA mediates the tumor-suppressive property of KLF6 through KLF6's anti-cancer pathways.

Several studies have reported that KLF6-SV1 regulated cancer cell cycle and survival, and it was shown that the targeted reduction of KLF6-SV1, using siRNA, induced apoptosis in lung cancer [10]. Here, we showed that the depletion of KLF6-SV1 by RNAi also promoted apoptosis, in gastric cancer cells in vitro, in a caspase-dependent manner. It is well known that apoptosis in mammalian cells is regulated by two major pathways, one involving the mitochondria (intrinsic pathway) and the other involving death receptors (extrinsic pathway) [38]. The overall integrity of the mitochondrial function is controlled by the Bcl-2 family of proteins that includes both anti-apoptotic (Bcl-2, Bcl-XL, and Mcl-1) and proapoptotic members (Bax, Bak, Noxa, and p53 upregulated modulator of apoptosis (PUMA)) [31, 32, 39-41]. Previous reports have demonstrated that the targeted reduction of KLF6-SV1 resulted in marked activation of the intrinsic pathway of apoptosis through upregulation of the pro-apoptotic Noxa and degradation of the anti-apoptotic Mcl-1 in lung, prostate, and ovarian cancer cell lines [10, 24, 26]. Here, we also confirmed that the expressions of Noxa and Mcl-1 could be regulated by KLF6-SV1 siRNA in gastric cancer cell lines. Moreover, other Bcl-2 related-apoptotic proteins, such as Bim and Bak, were also involved in the KLF6-SV1 siRNA-induced apoptosis in gastric cancer cell lines. Interestingly, in the present study, a novel apoptotic signaling regulation by KLF6-SV1 was observed. We found that KLF6-SV1 silencing downregulated the phosphorylation of Akt, which is a common mediator of cell survival signals, and has been shown to inhibit apoptosis by attenuating the activity of the pro-apoptotic factors Bad and caspase- 9 , and enhance survival by increasing the phosphorylation of $\mathrm{I} \kappa \mathrm{B}$ kinase (IKK) $\beta$, which led to the activation of nuclear factor (NF)- $\kappa \mathrm{B}$ [42]. In addition, Akt activation also could inhibit tumor apoptosis through the negative regulation of forkhead (Drosophila) homolog 1 (rhabdomyosarcoma) (FKHR), a member of the forkhead family of transcription factors, which could promote apoptosis by inducing the expression of Bim and FasL [43]. Thus, taking into consideration these combined findings, we suggest that KLF6-SV1 silencing inhibited Akt phosphorylation, and regulated the balance between the proapoptotic protein Noxa and the antiapoptotic proteins Bcl-xL and Mcl-1, inducing the dysfunction of mitochondria, which led to the caspase-dependent death of gastric cancer cells. Of course, although changes in apoptosis related-signaling in several proteins were determined, the precise mechanisms need more extensive study.

Our in vitro and in vivo data represent the first report describing mechanistic evidence for KLF6-SV1 as a mediator of the growth, migration, invasion, and survival of gastric cancer, using gastric cancer cell lines, and the evidence suggests that KLF6-SV1 is a potential therapeutic target for gastric tumors. A number of recent studies have highlighted the potential efficacy and specificity of siRNA/ RNAi-based therapies in mouse and/or non-human primate models of a number of human disorders ranging from respiratory syncytial virus (RSV) infection [44] to hypercholesteremia [45]. Beyond animal models, a number of RNAi therapeutic agents have already been in clinical trials and their number is increasing steadily [46]. Prospects for the treatment of cancer with these agents have also been recognized [47]. However, the major obstacle for moving gene silencing from the bench to the bedside remains the issue of delivery. Theoretically, targeting those disorders wherein the siRNA can be delivered directly into the diseased tissue would represent the most attractive option. The direct delivery route ensures a high concentration of the siRNA and would be predicted to have the least likelihood of unanticipated effects systemically. PEI, a readily available synthetic polycation introduced for transfection a few years ago [48], is an ideal candidate for gene delivery owing to its relatively high transfection efficiency and low toxicity. Therefore, we elected to use PEI as the KLF6-SV1 siRNA vector delivery system, and found that the intratumoral administration of PEI/si-SV1 effectively suppressed tumor growth and prolonged animal survival. Importantly, no adverse effects were observed (data not shown).

In the present study, we merely demonstrate that KLF6SV1 downregulation suppresses the tumorigenicity of gastric cancer cell lines. In the nude mice experiments in this study, permanent gastric cancer cell lines were exclusively used. Thus, the effect of KLF6-SV1 siRNA on human gastric cancer specimens and freshly isolated primary human gastric cancer cells needs further investigation in future experiments.

Acknowledgments This work was supported by grants from the Special Funds for Natural Science Foundation of Heilongjiang 
Province (D2007-69) and the Science and Technology Bureau Research Project in Harbin (2007AA3CS083 and 2007AA3CS083-1).

Conflict of interest The authors declare no competing financial interests.

\section{References}

1. Gordon DL. Tumors of the stomach. In: Feldman M, editor. Gastrointestinal and liver disease: pathophysiology, diagnosis, management. Philadelphia: WB Saunders; 2005. p. 733-57.

2. Roukos DH. Current status and future perspectives in gastric cancer management. Cancer Treat Rev. 2000;26:243-55.

3. Ushijima T, Sasako M. Focus on gastric cancer. Cancer Cell. 2004:5:121-5.

4. Black AR, Black JD, Azizkhan-Clifford J. Sp1 and Kruppel-like factor family of transcription factors in cell growth regulation and cancer. J Cell Physiol. 2001;188:143-60.

5. Narla G, Heath KE, Reeves HL, Li D, Giono LE, Kimmelman AC, et al. KLF6, a candidate tumour suppressor gene mutated in prostate cancer. Science. 2001;294:2563-6.

6. Chen C, Hyytinen ER, Sun X, Helin HJ, Koivisto PA Jr, Frierson $\mathrm{HF}$, et al. Deletion, mutation, and loss of expression of KLF6 in human prostate cancer. Am J Pathol. 2003;162:1349-54.

7. Reeves HL, Narla G, Ogunbiyi O, Haq AI, Katz A, Benzeno S, et al. Kruppel-like factor 6 (KLF6) is a tumour suppressor gene frequently inactivated in colorectal cancer. Gastroenterology. 2004;126:1090-3.

8. Cho YG, Choi BJ, Kim CJ, Song JW, Kim SY, Nam SW, et al. Genetic alterations of the KLF6 gene in colorectal cancers. APMIS. 2006;114:458-64.

9. Ito G, Uchiyama M, Kondo M, Usami SMN, Maeda O, Kawabe $\mathrm{T}$, et al. Kruppel-like factor 6 is frequently down-regulated and induces apoptosis in nonsmall cell lung cancer cells. Cancer Res. 2004;64:3838-43.

10. DiFeo A, Feld L, Rodriguez E, Wang C, Beer DG, Martignetti JA, et al. A functional role for KLF6-SV1 in lung adenocarcinoma prognosis and chemotherapy response. Cancer Res. 2008;68:965-70.

11. Cho YG, Kim CJ, Park CH, Yang YM, Kim SY, Nam SW, et al. Genetic alterations of the KLF6 gene in gastric cancer. Oncogene. 2005;24:4588-90.

12. Jeng YM, Hsu HC. KLF6, a putative tumour suppressor gene, is mutated in astrocytic gliomas. Int J Cancer. 2003;105:625-9.

13. Camacho-Vanegas O, Narla G, Teixeira MS, DiFeo A, Misra A, Singh $\mathrm{G}$, et al. Functional inactivation of the KLF6 tumour suppressor gene by loss of heterozygosity and increased alternative splicing in glioblastoma. Int J Cancer. 2007;121:1390-5.

14. Chen HK, Liu XQ, Lin J, Chen TY, Feng QS, Zeng YX. Mutation analysis of KLF6 gene in human nasopharyngeal carcinomas. Ai Zheng. 2002;21:1047-50.

15. Kremer-Tal S, Reeves HL, Narla G, Thung SN, Schwartz M, Difeo A, et al. Frequent inactivation of the tumour suppressor Kruppel-like factor 6 (KLF6) in hepatocellular carcinoma. Hepatology. 2004;40:1047-52.

16. Pan XC, Chen Z, Chen F, Chen XH, Jin HY, Xu XY. Inactivation of the tumour suppressor Kruppel-like factor 6 (KLF6) by mutation or decreased expression in hepatocellular carcinomas. J Zhejiang Univ Sci B. 2006;7:830-6.

17. Song J, Kim CJ, Cho YG, Kim SY, Nam SW, Lee SH, et al. Genetic and epigenetic alterations of the KLF6 gene in hepatocellular carcinoma. J Gastroenterol Hepatol. 2006;21:1286-9.
18. Kremer-Tal S, Narla G, Chen Y, Hod E, DiFeo A, Yea S, et al. Downregulation of KLF6 is an early event in hepatocarcinogenesis, and stimulates proliferation while reducing differentiation. J Hepatol. 2007;46:645-54.

19. Hartela M, Narla G, Wente MN, Giese NA, Martignoni ME, Martignetti JA, et al. Increased alternative splicing of the KLF6 tumour suppressor gene correlates with prognosis and tumour grade in patients with pancreatic cancer. Eur $\mathbf{J}$ Cancer. 2008;44:1895-903.

20. DiFeo A, Narla G, Hirshfeld J, Camacho-Vanegas O, Narla J, Rose SL, et al. Role of KLF6 and KLF6-SV1 in ovarian cancer progression and intraperitoneal dissemination. Clin Cancer Res. 2006;12:3730-9.

21. Narla G, DiFeo A, Reeves HL, Schaid DJ, Hirshfeld J, Hod E, et al. A germline DNA polymorphism enhances alternative splicing of the KLF6 tumor suppressor gene and is associated with increased prostate cancer risk. Cancer Res. 2005;65: 1213-22.

22. Teixeira MS, Camacho-Vanegas O, Fernandez Y, Narla G, DiFeo A, Lee B, et al. KLF6 allelic loss is associated with tumor recurrence and markedly decreased survival in head and neck squamous cell carcinoma. Int J Cancer. 2007;121:1976-83.

23. Narla G, DiFeo A, Yao S, Banno A, Hod E, Reeves HL, et al. Targeted inhibition of the KLF6 splice variant, KLF6 SV1, suppresses prostate cancer cell growth and spread. Cancer Res. 2005;65:5761-8.

24. Narla G, DiFeo A, Fernandez Y, Dhanasekaran S, Huang F, Sangodkar J, et al. KLF-SV1 overexpression accelerates human and mouse prostate cancer progression and metastasis. J Clin Invest. 2008;118:2711-21.

25. Sangodkar J, Shi J, DiFeo A, Schwartz R, Bromberg R, Choudhri A, et al. Functional role of the KLF6 tumour suppressor gene in gastric cancer. Eur J Cancer. 2009;45:666-76.

26. Difeo A, Huang F, Sangodkar J, Terzo EA, Leake D, Narla G, et al. KLF6-SV1 is a novel antiapoptotic protein that targets the BH3-only protein NOXA for degradation and whose inhibition extends survival in an ovarian cancer model. Cancer Res. 2009;69:4733-41.

27. Fan KX, Dai JX, Wang H, Wei HF, Cao ZG, Hou S, et al. Treatment of collagen-induced arthritis with an anti-osteopontin monoclonal antibody through promotion of apoptosis of both murine and human activated T cells. Arthritis Rheum. 2008;58: 2041-52.

28. Mehlen P, Puisieux A. Metastasis: a question of life or death. Nat Rev Cancer. 2006;6:449-58.

29. Igney FH, Krammer PH. Death and anti-death: tumour resistance to apoptosis. Nat Rev Cancer. 2002;2:277-88.

30. DiFeo A, Martignetti JA, Narla G. The role of KLF6 and its splice variants in cancer therapy. Drug Resist Updat. 2009;12:1-7.

31. Gross A, McDonnell JM, Korsmeyer SJ. BCL-2 family members and the mitochondria in apoptosis. Genes Dev. 1999;13: 1899-911.

32. Kroemer G. Mitochondrial control of apoptosis: an overview. Biochem Soc Symp. 1999;66:1-15.

33. Zha J, Harada H, Osipov K, Jockel J, Waksman G, Korsmeyer SJ. Serine phosphorylation of death agonist BAD in response to survival factor results in binding to 14-3-3 not Bcl-xl. Cell. 1996;87:619-28.

34. Wang J, Yang J, Kuropatwinski K, Wang W, Liu XQ, Hauser J, et al. Transforming growth factor beta induces apoptosis through repressing the phosphoinositide 3-kinase/AKT/survivin pathway in colon cancer cells. Cancer Res. 2008;68:3152-60.

35. Jia SF, Worth LL, Densmore CL, Xu B, Zhou Z, Kleinerman ES. Eradication of osteosarcoma lung metastases following intranasal 
interleukin-12 gene therapy using a nonviral polyethylenimine vector. Cancer Gene Ther. 2002;9:260-6.

36. Benzeno S, Narla G, Allina J, Cheng GZ, Reeves HL, Banck MS, et al. Cyclin dependent kinase inhibition by the KLF6 tumor suppressor protein through interaction with cyclin D1. Cancer Res. 2004;64:3885-91.

37. Slavin DA, Koritschoner NP, Prieto CC, Lopez-Diaz FJ, Chatton B, Bocco JL. A new role for the Kruppel-like transcription factor KLF6 as an inhibitor of c-jun proto-oncoprotein function. Oncogene. 2004;23:8196-205.

38. Ashkenazi A, Dixit VM. Death receptors: signaling and modulation. Science. 1998;281:1305-8.

39. Bartke T, Siegmond D, Peters N, Reichwein M, Henkler F, Scheurich $\mathrm{P}$, et al. p53 upregulates cFLIP, inhibits transcription of NF-kappaB-regulated genes and induces caspase-8-independent cell death in DLD-1 cells. Oncogene. 2001;20:571-80.

40. Yu J, Zhang L, Hwang PM, Kinzler KW, Vogelstein B. PUMA induces the rapid apoptosis of colorectal cancer cells. Mol Cell. 2001;20:812-8.

41. Zhang L, Ming L, Yu J. BH3mimetics to improve cancer therapy: mechanisms and examples. Drug Resist Updat. 2007;10:207-17.

42. Jeong SJ, Pise-Masison CA, Radonovich MF, Park HU, Brady JN. Activated AKT regulates NF-kappaB activation, p53 inhibition and cell survival in HTLV-1-transformed cells. Oncogene. 2005;24:6719-28.

43. Tang ED, Nuñez G, Barr FG, Guan KL. Negative regulation of the forkhead transcription factor FKHR by Akt. J Biol Chem. 1999;274:16741-6.

44. Bitko V, Musiyenko A, Shulyayeva O, Barik S. Inhibition of respiratory viruses by nasally administered siRNA. Nat Med. 2005;11:50-5.

45. Frank-Kamenetsky M, Grefhorst A, Anderson NN, Racie TS, Bramlage B, Akinc A, et al. Therapeutic RNAi targeting PCSK9 acutely lowers plasma cholesterol in rodents and LDL cholesterol in nonhuman primates. Proc Natl Acad Sci USA. 2008;105: 11915-20.

46. de Fougerolles A, Vornlocher HP, Maraganore J, Lieberman J. Interfering with disease: a progress report on siRNA based therapeutics. Nat Rev Drug Discov. 2007;6:443-53.

47. Pai SI, Lin YY, Macaes B, Meneshian A, Hung CF, Wu TC. Prospects of RNA interference therapy for cancer. Gene Ther. 2006;13:464-77.

48. Boussif O, Lezoualc'h F, Zanta A, Mergny MD, Scherman D, Demeneix B, et al. A versatile vector for gene and oligonucleotide transfer into cells in culture and in vivo: polyethylenimine. Proc Natl Acad Sci USA. 1995;92:7297-301. 